\title{
Hypothesis on the glucose production's communication model between the brain and other internal organs, especially the stomach and liver
}

Gerald C. Hsu

eclaireMD Foundation, USA

\author{
Correspondence author \\ Gerald C. Hsu \\ EclaireMD Foundation \\ USA
}

Submitted : 02 June 2020 ; Published : 18 June 2020

\begin{abstract}
After reviewing the research results for six months, from September 2019 through February 2020, the author identified a probable internal communication model between the nervous system and certain vital internal organs, specifically the stomach and liver regarding postprandial plasma glucose (PPG) production. The author used a continuous glucose monitor device to collect 50,000 glucose data during the past 665 days. He focused on studying the relationships among different food nutritional contents, cooking methods, food material's physical phases, and different characteristics and variants from his glucose waveform patterns. In this study, he focused on the three major meal groups based on food nutritional ingredients, meal's preparation, and cooking methods of eggs, squash, and cabbage to create soup-based (liquid) meal and pan-fried (solid) meal. The PPG waveforms from these three meal groups demonstrated that soup-based liquid food produced a much lower glucose value than the pan-fried solid food. Although both liquid and solid meals have similar identical nutritional ingredients, he questions why did this occur? His hypothesis is that his PPG differences are due to specific physical phase of his finished meal either "liquid" or "solid", which is his ready-to-eat meal's final physical "phase" that determines his PPG characteristics and waveforms. The author utilized his GH-Method: math-physical medicine (MPM) approach to explore a T2D patient's glucose production situation from a scientific view of the brain and nervous system's functionalities. If this specific approach and above interpretation are accurate, we can then "trick" our brain into producing a "lesser" amount of glucose after food intake without altering or sacrificing the needed food nutritional balance. As a result, T2D patients can simply change their cooking method in order to lower both of their peak $P P G$ values and their average PPG levels.
\end{abstract}

\section{Introduction}

In this paper, the author presents his 6-month research results, from September 2019 through February 2020, to identify a probable internal communication model between the brain nervous system and certain vital internal organs, specifically the stomach and liver regarding postprandial plasma glucose (PPG) production.

\section{Method}

The author used a continuous glucose monitor (CGM) device to collect 50,000 glucose data during the past 665 days (from $5 / 5 / 2018$ through $2 / 26 / 2020$ on the average of 75.53 glucose measurements per day). After the first bite of his meal, he measured his PPG data every 15 minutes for a 3-hour period (180 minutes). In addition, he also measured his PPG value at 2-hours after his meal via the finger-piercing method. He focused on studying the relationships among different food nutritional contents, cooking methods, food material's physical phases, and different characteristics and variants from his glucose waveform patterns (i.e. PPG curves). Based on his careful observations of physical phenomena of glucose results, he has some bold hypotheses for a probable communication model among the brain, stomach, liver and pancreas via our nervous system regarding glucose production amount, timing, and pattern. He then attempted to verify his created hypotheses via his big data analytics from his collected thousands of data based on food and glucose along with certain appropriate mathematical and AI tools.

In this particular study, he focused on the following three major meal groups. Figures 1, 2 and 3 have shown food nutritional ingredients, meal's preparation, and cooking methods of eggs, squash, and cabbage to create soup-based (liquid) meal and pan-fried (solid) meal. 


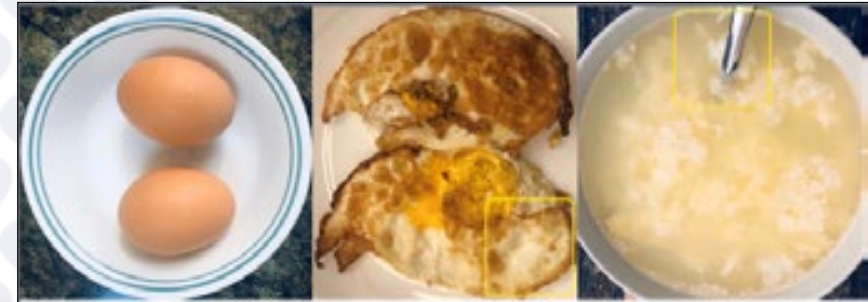

Egg (Whole)

1 large * RDI $3 \%-74 \mathrm{kcal}$

Serving Size 1 large

Calories: 74

(Calories from Fat: 45)

Total Fat: $4.97 \mathrm{~g}$

Total Carb: $0.38 \mathrm{~g}$

(Saturated Fat: $1.55 \mathrm{~g}$ )

Protein: $6.29 \mathrm{~g}$

Figure 1: Egg (liquid \& solid)

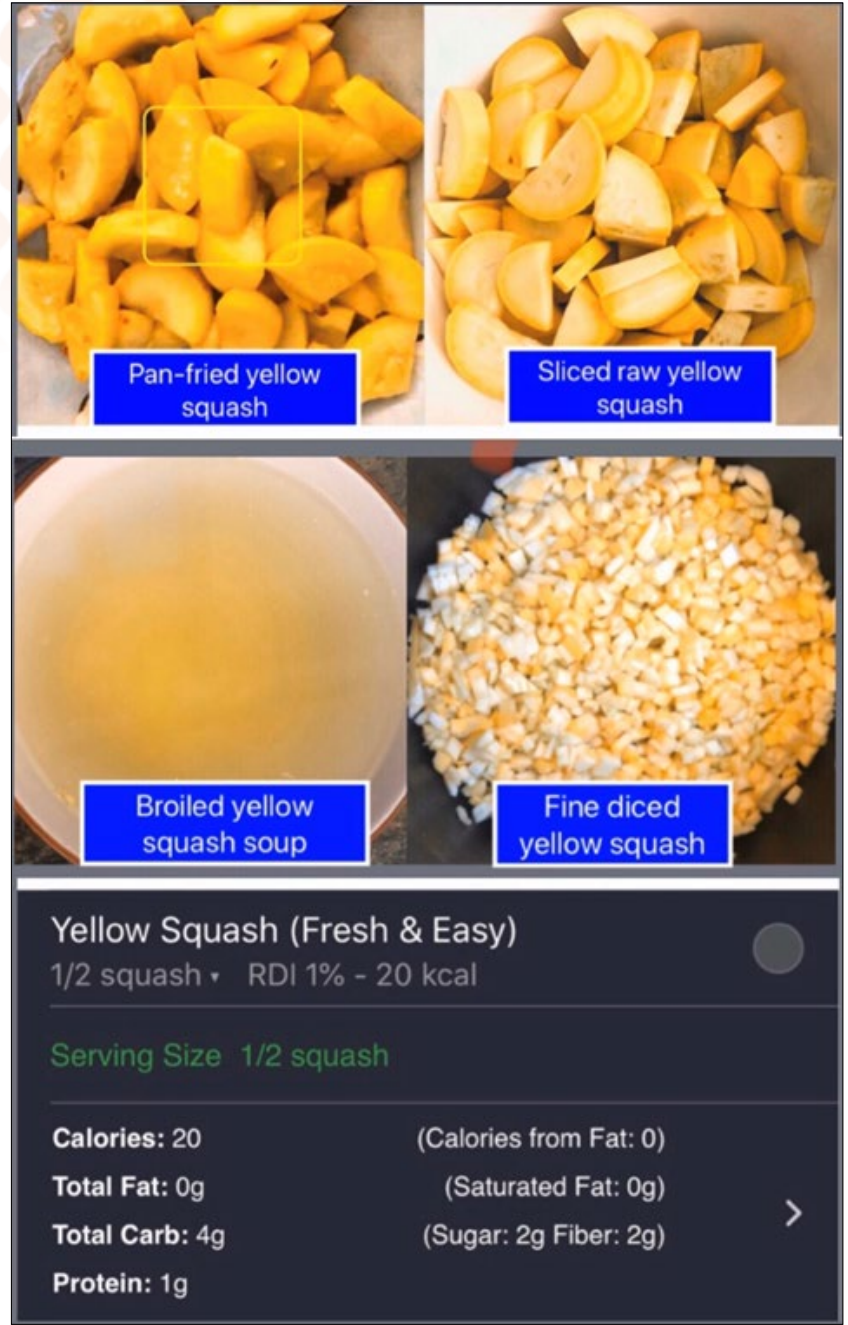

Figure 2: Squash (liquid \& solid)

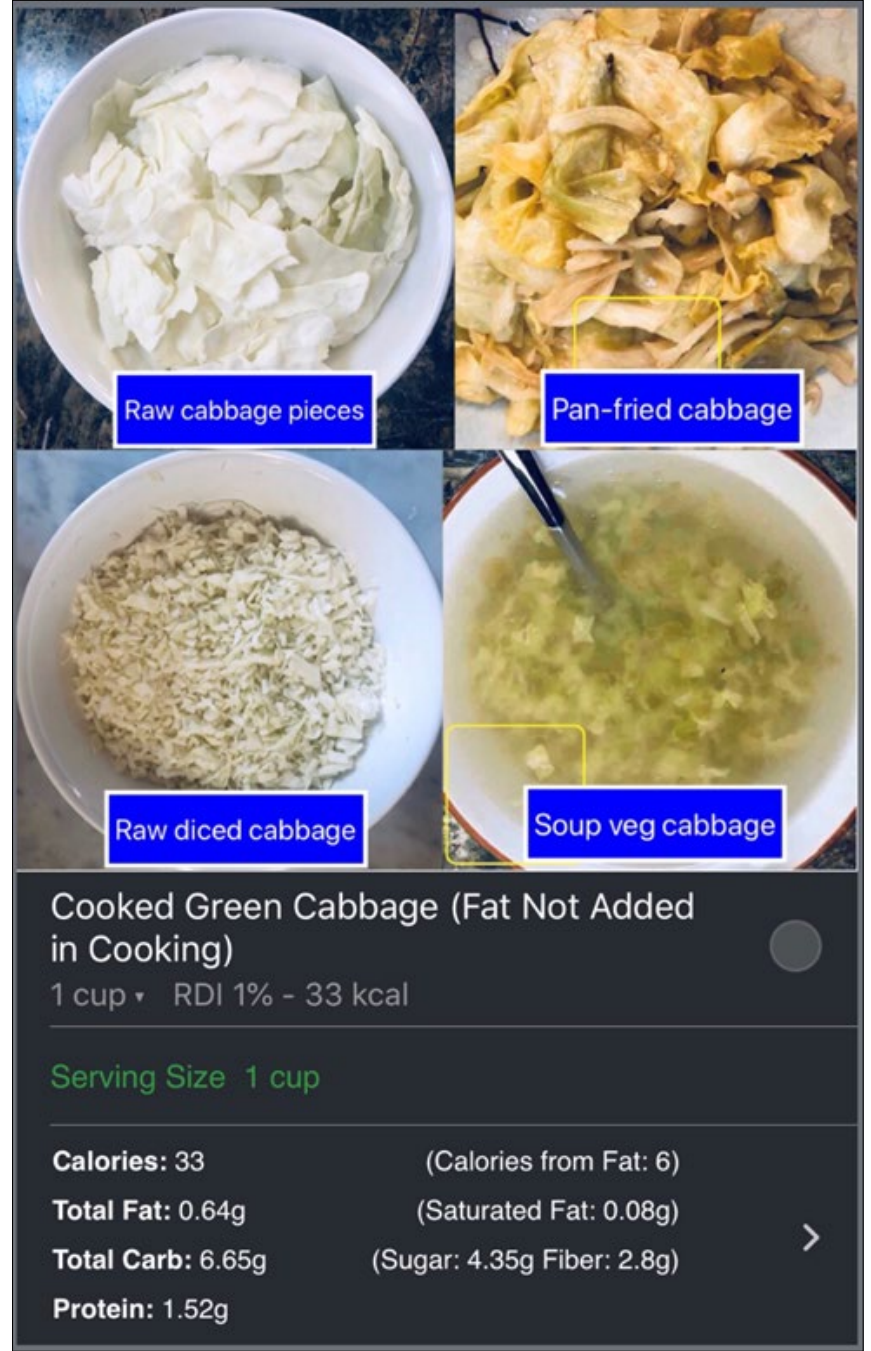

Figure 3: Cabbage (liquid \& solid)

In the first group, he had 46 soup-based, i.e. broth style of "liquid" phase meals which consist of 30 egg meals, 8 squash meals, and 8 cabbage meals. In this study, "broth" means without chunky pieces.

In the second group, he had 46 pan-fried, "solid" phase meals which also consist of 30 egg meals, 8 squash meals, and 8 cabbage meals.

In the above first two meal groups, pan-fried vegetables were cut into $\sim 58$ square centimeters ( $\sim 9$ square inches) for cabbage and $\sim 14.5$ square centimeters ( $\sim 2.25$ square inches) for squash. Small amount of olive oil and salt (both have zero carbs/sugar) were used to cook the pan-fried solid food. However, soupbased vegetables were diced into many tiny pieces and then boiled in hot water for $\sim 10$ minutes to reach to a liquified broth. For egg drop soup, he poured two mixed eggs into boiling water slowly to turn the eggs into thin-layered "clouds or sheets".

Both liquid and solid meals have the same food nutritional ingredients and almost identical amounts. For example, the "liquid" phase of egg drop soup contains the exact same nutritional ingredients and amounts as pan-fried eggs. Both 
of them used two large organic eggs that contains only protein (13 grams in egg white), fat (10 grams in egg yolk), and a tiny amount of carbohydrates ( 0.8 gram).

The third meal group contains a total of 2,048 meals during the past 665 days $(5 / 5 / 2018-2 / 26 / 2020)$, which includes a variety of meals eaten in more than 50 international cities.

\section{Results}

Figure 4 shows detailed data of sub-group meals (egg, squash, cabbage) such as carbs/sugar amount in grams, post-meal walking steps, PPG values in $\mathrm{mg} / \mathrm{dL}$ using both finger-piercing and sensor-collected methods.

\begin{tabular}{|l|c|c|c|c||}
\hline \hline Sensor PPG & Egg drop soup & Pan-fried egg & Soup veg & Pan-fried veg \\
\hline \hline \# of Meals & 30 & 30 & 16 & 16 \\
\hline Carbs grams & 0.8 & 0.7 & 11.0 & 12.8 \\
\hline Post-bkfst walk steps & 4843 & 4679 & 5175 & 4685 \\
\hline Avg Finger PPG & 108 & 115 & 108 & 111 \\
\hline Avg Sensor PPG & 117 & 139 & 111 & 123 \\
\hline PPG \& \% of PPG > 140 & $0(0 \%)$ & $147(46 \%)$ & $0(0 \%)$ & $140(15 \%)$ \\
\hline PPG difference (Peak-Start) & 4 & 22 & 7 & 20 \\
\hline PPG Peak time & 45 min & 75 min & 30 min & 60 min \\
\hline \hline Sensor PPG & Egg drop soup & Pan-fried egg & Soup veg & Pan-fried veg \\
\hline 0 min & 116 & 130 & 112 & 120 \\
\hline $15 \mathrm{~min}$ & 118 & 135 & 116 & 120 \\
\hline $30 \mathrm{mim}$ & 119 & 142 & 119 & 129 \\
\hline $45 \mathrm{~min}$ & 120 & 150 & 117 & 140 \\
\hline $60 \mathrm{~min}$ & 118 & 151 & 115 & 141 \\
\hline $75 \mathrm{~min}$ & 115 & 152 & 111 & 134 \\
\hline $90 \mathrm{~min}$ & 115 & 145 & 109 & 124 \\
\hline $105 \mathrm{~min}$ & 115 & 141 & 107 & 119 \\
\hline $120 \mathrm{~min}$ & 114 & 134 & 109 & 114 \\
\hline $135 \mathrm{~min}$ & 114 & 133 & 109 & 113 \\
\hline $150 \mathrm{~min}$ & 116 & 132 & 108 & 114 \\
\hline $165 \mathrm{~min}$ & 120 & 133 & 105 & 115 \\
\hline $180 \mathrm{~min}$ & 121 & 132 & 104 & \\
\hline
\end{tabular}

Figure 4: Data of egg, cabbage, squash

Summarized findings are:

1. Carbs/sugar amount: optical physics AI guesstimated carbs/sugar amount: eggs 0.7-0.8 gram, vegetables 11-15 grams, which matches with his acquired knowledge from his 9 -years learning on food nutrition.

2. Post-meal walking: all walks are more than 4,000 steps $(4,400-5,300)$ which means that this particular research can ignore the exercise influence on PPG. It should be noted that his body weight has been maintained in the range of 170-174 lbs. (77-79 kg).

3. Finger-piercing PPG: its range is between $105-115 \mathrm{mg} / \mathrm{dL}$ which indicates that the traditional finger-piercing testing results at 2-hours after the first bite of meal are not able to derive any meaningful findings in this research area. His particular brain and nervous system research work requires a complete glucose waveform information in order to derive meaningful and useful results.

\begin{tabular}{|c|c|c|c|c|c|c|}
\hline Sensor PPG & Egg drop soup & Pan-fried egg & Soup veg cabbage & Pan-fried veg cabbage & Soup veg squash & Pan-fried veg squash \\
\hline I of Meals & 30 & 30 & \begin{tabular}{|c|}
8 \\
8
\end{tabular} & 8 & 8 & 8 \\
\hline Carbs grams & 0.8 & 0.7 & 11.1 & 14.8 & 110 & 11.4 \\
\hline Post-biktst walk steps & 4843 & 4679 & 5009 & 4960 & 5342 & 4411 \\
\hline Avg Finger PPG & 108 & 115 & 109 & 111 & 105 & 109 \\
\hline Avg Sensor PPG & 117 & 139 & 112 & 120 & 110 & 126 \\
\hline$P P G \& \&$ of $P P G>140$ & $0(0 x)$ & $147(46 \times)$ & $0(0 x)$ & $0(0 x)$ & $0(0 x)$ & $146(15 \mathrm{X})$ \\
\hline PPG difference (Peak-Start) & 4 & 22 & 6 & 12 & 10 & 28 \\
\hline PPG Peak time & $45 \mathrm{~min}$ & $75 \mathrm{~min}$ & $45 \mathrm{~min}$ & $60 \mathrm{~min}$ & $30 \min$ & $60 \mathrm{~min}$ \\
\hline Sensor PPG & Egg drop soup & Pan-fried egg & Soup veg cabbage & Pan-fried veg cabbage & Soup veg squash & Pan-fried veg squash \\
\hline $0 \min$ & 116 & 130 & \begin{tabular}{|c|}
115 \\
\end{tabular} & 123 & 110 & \begin{tabular}{|c|}
118 \\
\end{tabular} \\
\hline $15 \mathrm{~min}$ & 118 & 135 & 116 & 122 & 116 & 119 \\
\hline $30 \mathrm{mim}$ & 119 & 142 & 119 & 129 & 120 & 129 \\
\hline $45 \mathrm{~min}$ & 120 & 150 & 121 & 135 & 114 & 145 \\
\hline $60 \mathrm{~min}$ & 118 & 151 & 119 & 135 & 112 & 146 \\
\hline $75 \mathrm{~min}$ & 115 & 152 & 111 & 133 & 111 & 135 \\
\hline $90 \mathrm{~min}$ & 115 & 145 & 108 & 121 & 109 & 127 \\
\hline $105 \mathrm{~min}$ & 115 & 141 & 105 & 115 & 110 & 123 \\
\hline $120 \mathrm{~min}$ & 114 & 134 & 105 & 110 & 112 & 119 \\
\hline $135 \mathrm{~min}$ & 114 & 133 & 110 & 107 & 108 & 121 \\
\hline $150 \mathrm{~min}$ & 116 & 132 & 108 & 110 & 108 & 116 \\
\hline $165 \mathrm{~min}$ & 120 & 133 & 107 & 111 & 104 & 117 \\
\hline $180 \mathrm{~min}$ & 121 & 132 & 111 & 112 & 98 & 118 \\
\hline
\end{tabular}

Figure 5: Data of egg \& vegetable 
Figure 5 shows data comparison between egg and vegetables (a combination of cabbage and squash) which includes both liquid and solid meals. The highlight of this data figure is that the "solid" meals have $15 \%$ to $46 \%$ of meals with PPG data $>140 \mathrm{mg} / \mathrm{dL}$ while "liquid" meals do not have any data $>140$ $\mathrm{mg} / \mathrm{dL}$.

\begin{tabular}{|c|c|c|c|}
\hline Sensor PPG & Soup-based & Pan-fried & All Meals \\
\hline I of Meals & 46 & 46 & 2048 \\
\hline Carbs grams & 4.3 & 4.9 & 14.4 \\
\hline Post-bkfst walk steps & 4958 & $46 \leqslant 1$ & 4298 \\
\hline Ave Finter PPG & 108 & 113 & 115 \\
\hline Ave Sensor PPG & 115 & 134 & 135 \\
\hline PPG \& $\times$ of PPG $>140$ & $0(0 x)$ & $143(23 X)$ & $143(23 \mathrm{X})$ \\
\hline PPG difference (Peak-Start) & 5 & 21 & 16 \\
\hline PPG Peak time & $45 \mathrm{~min}$ & $60 \mathrm{~min}$ & $60 \mathrm{mn}$ \\
\hline Sensor PPG & Soup-based & Pan-fried & All Meals \\
\hline $0 \mathrm{~min}$ & 115 & 127 & 129 \\
\hline $15 \mathrm{~min}$ & 117 & 130 & 132 \\
\hline $30 \mathrm{mim}$ & 119 & 138 & 138 \\
\hline $45 \mathrm{~min}$ & 119 & 146 & 143 \\
\hline $60 \mathrm{~min}$ & 117 & 147 & 144 \\
\hline $75 \mathrm{~min}$ & 113 & 146 & 142 \\
\hline $90 \mathrm{~min}$ & 113 & 138 & 138 \\
\hline $105 \min$ & 112 & 134 & 135 \\
\hline $120 \mathrm{~min}$ & 112 & 127 & 132 \\
\hline $135 \mathrm{~min}$ & 112 & 126 & 132 \\
\hline $150 \mathrm{~min}$ & 113 & 126 & 131 \\
\hline $165 \mathrm{~min}$ & 115 & 127 & 131 \\
\hline $180 \mathrm{~min}$ & 115 & 126 & 130 \\
\hline
\end{tabular}

Figure 6: Data of 46 soup-based (liquid) meals \& 46 pan-fried (solid) meals vs. 2048 All meals

Figure 6 shows comparison between solid and all meals versus liquid meals. It is obvious that two curves of 46 pan-fried solid food meals and all of 2,048 meals are clustered together. Both of them have a much higher value in both peak PPG and average PPG than the soup-based liquid food. It should be noted that different PPG waveforms would have different starting PPG values (at 0 -minute) due to his pre-meal food intake conditions, such as snack intakes, life stressors, or other physical conditions. Therefore, it is more logical to compare the PPG difference between peak PPG and starting PPG. The highlight of this PPG difference is that the solid meals have a PPG difference of $21 \mathrm{mg} / \mathrm{dL}$ and an average of $134 \mathrm{mg} / \mathrm{dL}$ and All meals have a PPG difference of $16 \mathrm{mg} / \mathrm{dL}$ and an average of $135 \mathrm{mg} / \mathrm{dL}$ in comparison with liquid meals have a PPG difference of $5 \mathrm{mg} / \mathrm{dL}$ and an average of $115 \mathrm{mg} / \mathrm{dL}$. Solid meals and all meals have 3 to 4 times higher PPG difference and $\sim 17 \%$ higher averaged PPG than liquid meals. Incidentally, the liquid meals averaged sensor PPG over three-hour period $(115 \mathrm{mg} / \mathrm{dL})$ is very close to finger-piercing PPG measured at two-hours after first bite of food $(\sim 110 \mathrm{mg} / \mathrm{dL})$.

PPG waveforms from these three meal groups in Figure 7 have demonstrated the final conclusive diagram of this 6-month research project. It is clear to observe that soup-based liquid food produced a much lower glucose value than the pan-fried solid food. Although both liquid and solid meals have similar identical nutritional ingredients, contrary to what he has learned about food nutrition, he questions why did this occur?

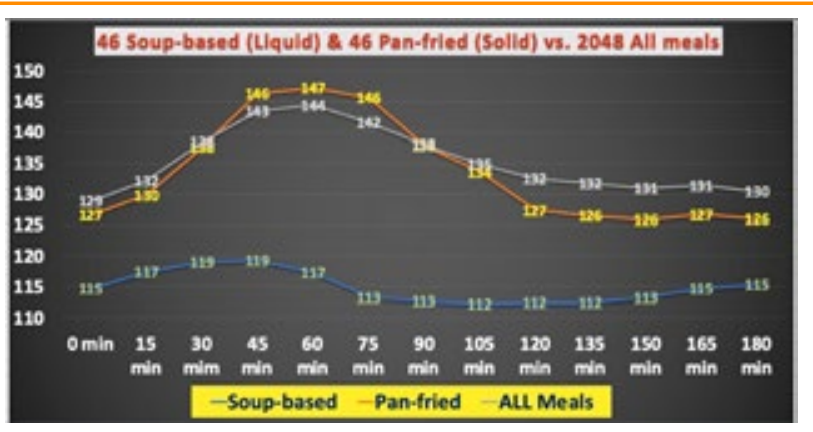

Figure 7: PPG wave comparison among 46 soup-based (liquid) meals \& 46 pan-fried (solid) meals vs. 2048 All meals

\section{Hypothesis}

During the past 9-years, the author have learned from both internal medicine and food nutrition that different glucose levels are converted from different carbs/sugar amount of food intake. However, what would be the reasonable explanation for these observed physical phenomenon of higher PPG values from solid food material and lower PPG values from liquid food material?

We have learned from physics in high school that there are three different fundamental phases of matter: solid, liquid, and gas (vapor or steam). This study of meals involves only two phases, liquid phase for both egg drop soup and vegetable (cabbage, squash) soup, and solid phase for the pan-fried eggs or vegetables. Of course, the group of all meals include both liquid phase and solid phase which were cooked in various cities and different dinning locations (with all of different kind of food materials).

The author has learned three basic facts from his past 9-years of medical research work. First, $\sim 70 \%$ of our daily energy intake are consumed by our brain and nervous system, not the heart as the author original thought. Second, the brain is the only internal organ which has the power of cognition, judgement, information processing, decision making, and marching order issuance like the CPU of a computer system. Third, all of our internal organs are working closely together in an integrated and very sophisticated manner but under the commands issued solely by our brain. The author has conducted a previous experiment of applying a special-designed IC chip on the skin of human leg and was able to identify the electronic flow and signal transmission via the nervous system between the brain and leg muscle. This experiment has also proved the specific function and mighty power of our brain.

Furthermore, the author also realized that glucose was produced by the liver via glycogen, but not "directly transformed" from food as indicated by many nutritionists and physicians. Glycogen is a substance deposited in bodily tissues to store carbohydrates. Initially, the liver breaks down most carbohydrates from the foods we eat and then converts them to a type of sugar called glucose. When the body doesn't need to use glucose for energy, it is stored in the liver and muscles. This stored form of glucose is made up of many connected glucose molecules (in a form of polysaccharide, not monosaccharide or 
oligosaccharide) known as glycogen.

The author's viewpoint toward this specific subject is based on his own different academic disciplines and professional trainings in the fields of mathematics, physics, engineering, and computer science. He believes the liver is an operating system which must have a "stimulator" (like food as an input), a "command center" (like the brain as a central processing unit), and a "feedback" (like glucose as an output). Therefore, glucose is not directly "converted" from food, but is rather directly "produced and released" by the liver, even though the liver needs food to supply as its "energy" source for its existence as well as its "raw material" for its glucose manufacturing capacities.

Based on the above acquired knowledge and his own interpretation, the author then developed his own hypothesis regarding glucose production by the liver. His hypothesis is that his PPG differences are due to specific physical phase of his meal, either "liquid" or "solid". The ready-to-eat meal's final physical "phase" determines the PPG characteristics and waveforms (i.e. both glucose height and curve shapes).

When one particular type of food enters into the gastrointestinal system, the stomach will immediately send a message to inform the brain regarding the food entry signal and its physical phase information. After receiving these input signals from the stomach, our brain will then process those related information, make judgements, and then issue the appropriate marching orders to our liver regarding how much glucose amount should be produced within what time frame. Obviously, this information processing and order-issuing action is a "dynamic" process since the biochemical conditions of food ingredients and human organ reactions are constantly changing with time. This is the reason time instants of reaching to the glucose peaks are different for different physical phase of food intake. At the same time, the brain will also inform our pancreas regarding how much insulin should be produced to offset the excessive amount of glucose generated by the liver. However, for diabetes patients whose pancreatic beta cells are severely damaged, their insulin production capabilities will not function properly.

For example, the author has observed from his 5,728 food and glucose data during the past 5 -years that his body usually takes about 10-15 minutes to reach to its glucose peak from high sugar content liquid food intake, about 45-60 minutes to reach to its glucose peak from liquid food intake, and about 60-75 minutes to reach to its glucose peak from solid food intake.

This particular hypothesis explains the author's view on how the brain communicates with the stomach, liver, and pancreas via our nervous system regarding the amount and pattern of PPG production during the 180-minute period after the first bite of meal. The reason for lower PPG values and a rather flat curve pattern associated with the liquid phase meals may be due to this specific food phase being able to "trick" the brain into thinking a similar food entry type such as drinking coffee, tea, or water; therefore, this may not cause an alert to increase the glucose production amount by the liver.

Thus far, the author has conducted his experiments on meals with "mono-material" only; however, most people prefer to have a meal with "multi-materials". Therefore, he will continue his experiments moving toward this direction. He also urges other T2D patients, who are using the CGM device, to conduct similar experiments in order to collect more glucose data from people with different DNA genes and diabetes conditions.

\section{Conclusion}

The author utilized his GH-Method: math-physical medicine (MPM) approach to explore a T2D patient's glucose production situation from a scientific view of the brain and nervous system's functionalities. If this specific approach and above interpretation are accurate, we can then "trick" our brain into producing a "less" amount of glucose after food intake without altering or sacrificing the needed food nutritional balance. As a result, T2D patients can simply change their cooking method in order to lower both of their peak PPG values and their average PPG levels. However, T2D patients must continually avoid eating food materials with high carbohydrates food such as starchy grains or flours or high sugar contents such as sweetened cakes or drinks.

By sharing his research findings with other fellow medical research scientists, he hopes that they can provide some other interpretations, and proper explanations, or offer further justifications to our entire medical and healthcare community by using a different or traditional research methodology, such as biochemical medicine (BCM) approach.

\section{References}

1. Hsu, Gerald C (2018) Using Math-Physical Medicine to Control T2D via Metabolism Monitoring and Glucose Predictions. Journal of Endocrinology and Diabetes 1(1): 1-6.

2. Hsu, Gerald C (2018) Using Signal Processing Techniques to Predict PPG for T2D. International Journal of Diabetes \& Metabolic Disorders 3(2): 1-3.

3. Hsu, Gerald C (2018) Using Math-Physical Medicine and Artificial Intelligence Technology to Manage Lifestyle and Control Metabolic Conditions of T2D. International Journal of Diabetes \& Its Complications 2(3): 1-7.

4. Hsu, Gerald C (2018) Using Math-Physical Medicine to Analyze Metabolism and Improve Health Conditions. Video presented at the meeting of the 3rd International Conference on Endocrinology and Metabolic Syndrome 2018, Amsterdam, Netherlands.

5. Hsu, Gerald C (2018) Using Math-Physical Medicine to Study the Risk Probability of having a Heart Attack or Stroke Based on Three Approaches, Medical Conditions, Lifestyle Management Details, and Metabolic Index. EC Cardiology 5(12): 1-9. 